PROCEEDINGS OF THE AMERICAN MATHEMATICAL SOCIETY

Volume 124, Number 7, July 1996

\title{
SUBALGEBRAS OF FREE ALGEBRAS
}

\author{
A. A. MIKHALEV, V. E. SHPILRAin, AND A. A. ZOLOTYKH \\ (Communicated by Lance W. Small)
}

\begin{abstract}
We use non-commutative Jacobian matrix to get information on finitely generated subalgebras of a free Lie algebra. In particular, we show that the rank of such a subalgebra is equal to the left rank (i.e., to the maximal number of left independent rows) of the corresponding Jacobian matrix; this also yields an effective procedure for finding the rank of a finitely generated subalgebra.
\end{abstract}

\section{INTRODUCTION}

In 1973, Birman [2] used a non-commutative analog of the Jacobian matrix to give a matrix characterization of automorphisms of a free group among arbitrary endomorphisms. About 20 years later, a series of results in different branches of algebra has led to important new applications of non-commutative Jacobian matrix. First of all, the analog of Birman's "inverse function theorem" has been proved for free Lie algebras in [13] and [15]; a very similar characterization is given in [9]. Then, Umirbaev [17] has generalized Birman's theorem to arbitrary (not necessarily square) Jacobian matrices over a free group ring; in [7] and [8], the corresponding result has been proved for free Lie algebras.

Essentially new properties of non-commutative Jacobian matrix have been discovered in [7], [14] and [18]. In [14], it is shown that, similar to the commutative situation, the rank of the subgroup of a free group generated by a finite set of elements, is equal to the maximal number of left independent rows of the corresponding Jacobian matrix. In this paper, we prove the analog of this result for free Lie algebras (Corollary 3.5) as a corollary of our main result - a matrix characterization of independent sets of elements (Theorem 3.4).

Yet another remarkable property of the (non-commutative) Jacobian matrix has been discovered first in [7] for matrices over free associative algebras, and then in [18] - for matrices over free group rings. It turns out that the columns of the Jacobian matrix are "responsible" for the outer rank of the corresponding set of elements of a free group [18] or a free Lie algebra [7]. Outer rank here is defined as the minimal rank of a free factor of a free group (or a free Lie algebra) that contains a given set of elements.

Received by the editors January 9, 1995.

1991 Mathematics Subject Classification. Primary 17B01; Secondary 16S10, 13F20.

The first and third authors were partially supported by the Russian Foundation for Fundamental Research, by the International Science Foundation, and by INTAS.

The second author was supported by MINERVA Fellowship.

(C)1996 American Mathematical Society 
These results reveal the following remarkable situation with the Jacobian matrix of a finite set of elements (this is not necessarily a square matrix):

(i) the invertibility of the Jacobian matrix (this is a property of matrix as a "whole entity") means that a given set of elements is (a part of) a free generating set;

(ii) the maximal number of left independent rows of the Jacobian matrix (this is a property of rows of a matrix) equals the rank of the subalgebra (subgroup) generated by a given set of elements;

(iii) the maximal number of right independent columns of the Jacobian matrix (this is a property of columns of a matrix) equals the outer rank of a given set of elements.

Although the study of non-commutative Jacobian matrices was originally motivated by similar issues in (commutative) polynomial algebras, property (i) in commutative case is not yet established for square Jacobian matrices (this is a notorious Jacobian conjecture — see e.g. [1] for a survey); for non-square matrices it just does not hold. Property (ii) in commutative situation (for polynomial algebras over a field of characteristic zero) is well-known (although non-trivial) - see e.g. [6, p. 192]. As regards property (iii), it just has not been studied in commutative situation to the best of our knowledge.

For elements of a free associative algebra, none of these properties seems to be valid - see Section 4 of this paper and [9]. We note however that it is possible to define partial derivations in a special enveloping object such that, for example, an analog of the Jacobian conjecture would hold for corresponding Jacobian matrices - see [4], [11].

For elements of a free group, all of these properties have been (recently) established.

In Section 3 of this paper, we complete this picture for elements of a free Lie algebra by proving property (ii) (properties (i) and (iii) have been already established - see above), and based on that, elaborate a convenient algorithm for finding the rank of the subalgebra generated by a given finite set of elements.

Although property (ii) does not in general hold for elements of a free associative algebra, we give some partial results in Section 4.

\section{Preliminaries}

By $L=L(X)$ we denote the free Lie algebra, and by $A=A(X)$ the free associative algebra over an arbitrary but fixed field $K$, where $X=\left\{x_{1}, \ldots, x_{n}\right\}, n \geq 2$, is a finite set of free generators. The algebra $L$ is naturally identified with a subalgebra of $A$, so we just assume $L \subseteq A$.

To define non-commutative Jacobian matrix, we have to introduce non-commutative partial derivatives first. We call them Fox derivatives in honor of R. Fox who considered them in a free group ring [5]. There is the augmentation homomorphism $\varepsilon: A \rightarrow K$ defined by $\varepsilon\left(x_{i}\right)=0,1 \leq i \leq n$. Its kernel $\Delta$ is called the augmentation ideal of $A$; it is a free left $A$-module with a free basis $X$, so that every element $u \in \Delta$ can be uniquely written in the form $u=\sum_{i=1}^{n} d_{i}(u) x_{i}$. The "coordinates" $d_{i}(u)$ of the element $u$ in the free basis $X$ are called (partial) Fox derivatives. We usually write here $\frac{\partial u}{\partial x_{i}}$ for $d_{i}(u)$.

One can extend these derivations linearly to the whole $A$ defining $d_{i}(1)=0$. 
Now for an arbitrary finite set of elements $Y=\left\{y_{1}, \ldots, y_{m}\right\} \subseteq A$, we can define the matrix

$$
J_{Y}=\left(\frac{\partial y_{i}}{\partial x_{j}}\right)_{1 \leq j \leq n, 1 \leq i \leq m},
$$

the Jacobian matrix of $Y$.

There is the "chain rule" for Fox derivatives (see [5]):

Lemma 2.1. Let $f$ be an endomorphism of $A$ defined by $\varphi\left(x_{k}\right)=y_{k}, 1 \leq k \leq n$, and let $f=f\left(x_{1}, \ldots, x_{n}\right)$ be an element of $\Delta$. Then

$$
\frac{\partial \varphi(f)}{\partial x_{j}}=\sum_{k=1}^{n} \frac{\partial f}{\partial y_{k}} \cdot \frac{\partial y_{k}}{\partial x_{j}}
$$

where by $\frac{\partial f}{\partial y_{k}}$ we mean $\varphi\left(\frac{\partial f}{\partial x_{k}}\right)$.

We also need a few notational agreements to be used in Section 3. We say that a set $Y \subseteq L$ is independent if $Y$ is a free generating set for the subalgebra it generates (in other words, there are no non-trivial relations between elements of $Y$ ).

Let $\ell$ be the usual length function on $A$. For an element $a \in A$, by $a^{\circ}$ we denote the sum of monomials of the highest degree in $a$ with respect to the function $\ell$. If $a=a^{\circ}$, then $a$ is a homogeneous element.

A set $Y \subseteq L$ is called reduced if for every $y \in Y$, the element $y^{\circ}$ does not belong to the subalgebra of $L$ generated by the set $\left\{u^{\circ} \mid u \in Y, u \neq y\right\}$.

Then we have a Shirshov's result [12]:

Theorem 2.2. Every reduced subset of $L$ is an independent set. Furthermore, every subalgebra of a free Lie algebra is free.

\section{Proof of the main Results} by $H$.

Let $H=\left\{h_{1}, \ldots, h_{m}\right\}$ be a subset of $L$, and $L(H)$ the subalgebra of $L$ generated

Lemma 3.1. For $a_{1}, \ldots, a_{m} \in A$, a matrix equality

$$
\left(a_{1}, \ldots, a_{m}\right) \cdot J_{H}=(0)
$$

holds if and only if

$$
a_{1} h_{1}+\cdots+a_{m} h_{m}=0 .
$$

Proof. From $a_{1} h_{1}+\cdots+a_{m} h_{m}=0$, it follows that

$$
\frac{\partial}{\partial x_{j}}\left(a_{1} h_{1}+\cdots+a_{m} h_{m}\right)=\sum_{i=1}^{m} a_{i} \frac{\partial h_{i}}{\partial x_{j}}=0
$$

for $j=1, \ldots, n$. The matrix equality follows.

On the other hand, suppose

$$
\left(a_{1}, \ldots, a_{m}\right) \cdot J_{H}=(0) .
$$

Then we get for $j=1, \ldots, n$ :

$$
0=\sum_{i=1}^{m} a_{i} \frac{\partial h_{i}}{\partial x_{j}}=\frac{\partial}{\partial x_{j}}\left(\sum_{i=1}^{m} a_{i} h_{i}\right) .
$$

Since all $h_{i}$ have zero constant term, this yields $a_{1} h_{1}+\cdots+a_{m} h_{m}=0$. 
Lemma 3.2. Let $Y=\left\{y_{1}, \ldots, y_{m}\right\}$ be a subset of $L ; L(Y)$ the free Lie algebra generated by $Y$; and

$$
a_{1} h_{1}+\cdots+a_{m} h_{m}=0 .
$$

Set $g_{m}=h_{m}+f\left(h_{1}, \ldots, h_{m-1}\right)$ for some $f\left(y_{1}, \ldots, y_{m-1}\right) \in L(Y)$, and $g_{i}=h_{i}$, $i \neq m$. Then

$$
b_{1} g_{1}+\cdots+b_{m} g_{m}=0
$$

where

$$
b_{i}=a_{i}-a_{m} \cdot \frac{\partial f}{\partial y_{i}}\left(g_{1}, \ldots, g_{m-1}\right) \quad \text { when } i \neq m ; \quad b_{m}=a_{m} .
$$

Proof. We write:

$$
\begin{array}{r}
0=a_{1} h_{1}+\cdots+a_{m} h_{m}=\sum_{i=1}^{m-1} a_{i} g_{i}+a_{m}\left(g_{m}-f\left(g_{1}, \ldots, g_{m-1}\right)\right) \\
=\sum_{i=1}^{m-1}\left(a_{i}-a_{m} \cdot \frac{\partial f}{\partial y_{i}}\left(g_{1}, \ldots, g_{m-1}\right)\right) \cdot g_{i}+a_{m} g_{m}
\end{array}
$$

The result follows.

Lemma 3.3. Let $h_{1}, \ldots, h_{m}$ be homogeneous elements of $L$ such that

$$
a_{1} h_{1}+\cdots+a_{m} h_{m}=0
$$

for $a_{1}, \ldots, a_{m} \in A$, some of them non-zero. Then there is a dependence relation

$$
f\left(h_{1}, \ldots, h_{m}\right)=0
$$

for some non-zero $f \in L$.

Proof. Without loss of generality, we can assume that $a_{1}, \ldots, a_{m}$ are homogeneous elements, and that $h_{1}, \ldots, h_{m}$ are linearly independent. Using P. M. Cohn's weak algorithm (see [3]), we see that for some $i$ (we just take $i=1$ ), there is the following relation:

$$
h_{1}=b_{2} h_{2}+\cdots+b_{m} h_{m}
$$

where $b_{2}, \ldots, b_{m}$ are homogeneous elements of $A$. We see that $h_{1}$ belongs to the left ideal of $A$ generated by $\left\{h_{2}, \ldots, h_{m}\right\}$. After repeatedly applying the PoincaréBirkhoff-Witt theorem, we conclude that in fact $h_{1}$ belongs to the Lie subalgebra generated by $\left\{h_{2}, \ldots, h_{m}\right\}$.

Note that Lemma 3.3 is a particular case of Theorem 2.3 in Chapter 2 of the book [10].

Theorem 3.4. A subset $H$ of the free Lie algebra $L$ is independent if and only if the rows of the matrix $J_{H}$ are left independent over the free associative algebra A.

Proof. 1) Let $Y=\left\{y_{1}, \ldots, y_{m}\right\}$. Suppose there is a non-zero element $f\left(y_{1}, \ldots, y_{m}\right)$ of the free Lie algebra $L(Y)$ such that $f\left(h_{1}, \ldots, h_{m}\right)=0$. Among all non-zero elements $f \in A(Y)$ with this property, we choose an element $f_{0}$ of the minimal length $\ell\left(f_{0}\right)$ with respect to $Y$. Since the elements $h_{1}, \ldots, h_{m}$ have zero constant term, and $f_{0}\left(h_{1}, \ldots, h_{m}\right)=0$, we see that the constant term of $f_{0}$ is equal to zero as well. 
Since $f_{0}\left(h_{1}, \ldots, h_{m}\right)=0$, we have by Lemma 2.1 :

$$
\sum_{i=1}^{m} \frac{\partial f_{0}}{\partial y_{i}}\left(h_{1}, \ldots, h_{m}\right) \cdot \frac{\partial h_{i}}{\partial x_{j}}=0 .
$$

Therefore

$$
\left(a_{1}, \ldots, a_{m}\right) \cdot J_{H}=(0),
$$

where

$$
a_{i}=\frac{\partial f_{0}}{\partial y_{i}}\left(h_{1}, \ldots, h_{m}\right)
$$

By means of contradiction, assume that $a_{i}=0$ for all $i$. Since $\ell\left(\frac{\partial f_{0}}{\partial y_{i}}\right)<\ell\left(f_{0}\right)$, this yields $\frac{\partial f_{0}}{\partial y_{i}}=0$ for all $i$. Since the constant term of $f_{0}$ is equal to zero, we get $f_{0}=0$, hence a contradiction. Thus there is a non-trivial left dependence between the rows of the Jacobian matrix.

2) Suppose we have a matrix equality

$$
\left(a_{1}, \ldots, a_{m}\right) \cdot J_{H}=(0) .
$$

By Lemma 3.1, this yields

$$
a_{1} h_{1}+\cdots+a_{m} h_{m}=0 .
$$

Then we have for some $j$ :

$$
h_{j}^{\circ}=f\left(h_{1}^{\circ}, \ldots, h_{j-1}^{\circ}, h_{j+1}^{\circ}, \ldots, h_{m}^{\circ}\right) .
$$

Consider a system of elements $\left\{g_{1}, \ldots, g_{m}\right\}$ such that

$$
g_{j}=h_{j}-f\left(h_{1}, \ldots, h_{j-1}, h_{j+1}, \ldots, h_{m}\right),
$$

and $g_{i}=h_{i}$ for all $i \neq j$ (i.e., we apply an elementary transformation to $H$ ). By Lemma 3.2 , we have then

$$
b_{1} g_{1}+\cdots+b_{m} g_{m}=0
$$

for $b_{1}, \ldots, b_{m} \in A$, some of them non-zero. Since $\ell\left(g_{j}\right)<\ell\left(h_{j}\right)$ when $j \neq i$ and $\ell\left(g_{i}\right)=\ell\left(h_{i}\right)$, in some step of this procedure we either get a zero element (this gives a nontrivial Lie dependence relation between the elements of $H$ ), or we come to a reduced subset $\left\{g_{1}, \ldots, g_{m}\right\}$ with a non-trivial relation of the form (1).

We are now going to show that the second possibility cannot actually occur. Consider the leading part of the element on the left-hand side of (1); we get a non-trivial homogeneous relation

$$
c_{1} g_{1}^{\circ}+\cdots+c_{m} g_{m}^{\circ}=0 .
$$

By Lemma 3.3, this implies

$$
f\left(g_{1}^{\circ}, \ldots, g_{m}^{\circ}\right)=0
$$

for some non-zero $f \in L$. Since the subset $\left\{g_{1}, \ldots, g_{m}\right\}$ is reduced, we have a contradiction with Theorem 2.2 .

Thus there is a non-trivial dependence relation between the elements of $H$, and this completes the proof. 
Corollary 3.5. The rank of the subalgebra of $L$ generated by a finite set $H$ is equal to the rank of the free left $A$-submodule of the free $A$-module $A^{n}$ generated by the rows of the matrix $J_{H}$, i.e., to the maximal number of left independent rows of the Jacobian matrix.

Proof. If the subset $H$ is not reduced, then using a finite number of elementary transformations, we can get a reduced subset $H^{\prime}$ which generates the same subalgebra $L(H)$. Each elementary transformation of $H$ induces elementary transformation of the rows of $J_{H}$ : if, for example, $h_{1}^{\prime}=h_{1}-f\left(h_{2}, \ldots, h_{n}\right)$, then

$$
\left(\frac{\partial h_{1}^{\prime}}{\partial x_{1}}, \ldots, \frac{\partial h_{1}^{\prime}}{\partial x_{n}}\right)=\left(\frac{\partial h_{1}}{\partial x_{1}}, \ldots, \frac{\partial h_{1}}{\partial x_{n}}\right)-\sum_{i=2}^{n} \frac{\partial f}{\partial y_{i}}\left(h_{2}, \ldots, h_{n}\right) \cdot\left(\frac{\partial h_{i}}{\partial x_{1}}, \ldots, \frac{\partial h_{i}}{\partial x_{n}}\right) .
$$

Hence the left rank of $J_{H^{\prime}}$ is equal to the left rank of $J_{H}$. Applying Theorems 2.2 and 3.4 completes the proof.

Corollary 3.5 gives a convenient algorithm for finding the rank of a finitely generated subalgebra of $L$. Indeed, by using elementary transformations of the rows of the Jacobian matrix, we can construct a basis of the above mentioned free left $A$-submodule of the free left $A$-module $A^{n}$, and compute its rank thereby.

We note that Shirshov's results [12] give another algorithm for finding the rank.

Corollary 3.6. Let $\varphi$ be an endomorphism of $L$. Then the rank of the free Lie algebra $\varphi(L)$ coincides with the rank of the free left $A$-submodule of the free $A$ module $A^{n}$ generated by the rows of the matrix

$$
J_{\varphi}=J_{\left\{\varphi\left(x_{1}\right), \ldots, \varphi\left(x_{n}\right)\right\}} .
$$

Corollary 3.7. An endomorphism $\varphi$ of $L$ is a monomorphism if and only if the rows of the matrix $J_{\varphi}$ are left independent over $A$.

Remark 3.8. The rank of the left $A$-module generated by the rows of the matrix $J_{\varphi}$ might be different from the rank of the right $A$-module generated by those rows.

Indeed, let $X=\{x, y, z\}, \varphi(x)=[[x, y], z], \varphi(y)=[x, y], \varphi(z)=z$. Then

$$
J_{\varphi}=\left(\begin{array}{ccc}
z y & -z x & x y-y x \\
-y & x & 0 \\
0 & 0 & 1
\end{array}\right) .
$$

The rank of the above mentioned left $A$-module equals 2 , and at the same time the rank of the right $A$-module equals 3 .

\section{Concluding Remarks}

We begin this section by saying that none of the properties (i)-(iii) of the Jacobian matrix that are listed in the Introduction, is valid when we consider finite sets of elements of a free associative algebra. There are simple examples of invertible Jacobian matrices which do not correspond to generating systems of a free associative algebra - see e.g. [9]. However the following fact is worth keeping in mind:

Proposition 4.1. The following three conditions are equivalent:

(a) a matrix $M=\left(a_{i j}\right)_{1<i, j \leq n}$ is invertible over $A$ (i.e., $M \in \mathrm{GL}_{n}(A)$ );

(b) the elements $y_{j}=\sum_{k=1}^{n} x_{k} a_{k j}, 1 \leq j \leq n$, generate the augmentation ideal $\Delta$ as a right ideal of $A$;

(c) the elements $y_{j}=\sum_{k=1}^{n} a_{j k} x_{k}, 1 \leq j \leq n$, generate the augmentation ideal $\Delta$ as a left ideal of $A$. 
Proof. Suppose $M$ is invertible over $A$; denote by $R_{x}$ the row matrix $\left(x_{1}, \ldots, x_{n}\right)$, and by $R_{y}$ the row matrix $\left(y_{1}, \ldots, y_{n}\right)$. Then $R_{x} M M^{-1}=R_{y} M^{-1}=R_{x}$ which means that $x_{1}, \ldots, x_{n}$ belong to the right ideal of $A$ generated by $y_{1}, \ldots, y_{n}$. Conversely, suppose we have $R_{y} B=R_{x}$ for some matrix $B$ over $A$. Then $R_{x} M B=R_{x}$, hence $M B=I$, the identity matrix, because $\left(x_{1}, \ldots, x_{n}\right)$ form a free basis of a free right $A$-module $\Delta$. This implies $M \in \mathrm{GL}_{n}(A)$. The equivalence of (a) and (c) is treated similarly.

Note that condition (b) or (c) of this proposition would imply that $\left\{y_{1}, \ldots, y_{n}\right\}$ is a generating system of $A$ were all these $y_{i}$ Lie elements — see [9] or [13]. If they are arbitrary elements of $A$, this is not necessarily the case.

Now for a free associative algebra $A$, denote by $A^{e}$ the tensor product $A \otimes_{K} A$ with the multiplication given by $(a \otimes b)(c \otimes d)=a c \otimes d b$. Let

$$
I_{A}=\bigoplus_{i=1}^{n}\left(A^{e}\right)_{i}
$$

be the direct sum of $n=|X|$ copies of $A^{e}$. The universal derivation of $A$ is the $K$-linear mapping $D: A \rightarrow I_{A}$ defined by $D\left(x_{i}\right)=(1 \otimes 1)_{i}, D(a b)=D(a) \cdot b+a \cdot D(b)$, $D(1)=0$. For any $a \in A$, the element $D(a)$ has a unique presentation in the form $D(a)=a_{1}+\cdots+a_{n}, a_{i} \in\left(A^{e}\right)_{i}$. The component $a_{i}=\frac{\partial a}{\partial x_{i}}$ is called the (universal) partial derivative of $a$ with respect to $x_{i}$.

Then an endomorphism $\varphi$ of $A$ is an automorphism if and only if the matrix

$$
\left(\frac{\partial \varphi\left(x_{i}\right)}{\partial x_{j}}\right)_{1 \leq i, j \leq n}
$$

is invertible over $A^{e}$ - see [4], [11].

Consider now property (ii) in the case of a free associative algebra. Along the same lines as in part (1) of the proof of Theorem 3.4, one can prove the following

Proposition 4.2. Let $Y=\left\{y_{1}, \ldots, y_{m}\right\}$ be a set of elements of a free associative algebra $A$. If the rows of the Jacobian matrix $J_{Y}$ are left independent over $A$, then $Y$ is an independent set of $A$.

Corollary 4.3. The (left) rank of the Jacobian matrix $J_{Y}$ of a finite set of elements $Y \subseteq A$ is not bigger than the rank of the subalgebra of A generated by $Y$.

It is well known that a free associative algebra (as well as a free Lie algebra) of a finite rank is Hopfian (see e.g. [11]), i.e., every onto endomorphism of $A$ is actually an automorphism. Now we can obtain this result as a corollary of Proposition 4.2. Indeed, suppose an endomorphism $\varphi$ which takes $X=\left\{x_{1}, \ldots, x_{n}\right\}$ to $Y=\left\{y_{1}, \ldots, y_{n}\right\}$, is onto; then by Lemma 2.1, the Jacobian matrix $J_{Y}$ is invertible, in particular its rows are left independent, hence by Proposition $4.2, \varphi$ is a monomorphism.

Remark 4.4. The converse of Proposition 4.2 does not hold. For example, for $x, y \in$ $X$ the elements $x$ and $y x$ generate a free subalgebra of $A$ whereas the rows of the corresponding Jacobian matrix are left dependent over $A$. Various necessary and sufficient conditions for a subalgebra of $A$ to be free can be found in [3]. However, Umirbaev [16] has recently proved that there is no algorithm for deciding whether or not a given finite set of elements of $A$ is an independent set. There is, on the 
other hand, a (rather complicated) algorithm for detecting free generating sets of the whole algebra $A-$ see [19].

\section{REFERENCES}

1. H. Bass, E. H. Connell, and D. Wright, The Jacobian conjecture: reduction of degree and formal expansion of the inverse. Bull. Amer. Math. Soc. (N. S.) 7 (1982), no. 2, 287-330. MR 83k: 14028

2. J. S. Birman, An inverse function theorem for free groups. Proc. Amer. Math. Soc. 41 (1973), 634-638. MR 48:8632

3. P. M. Cohn, Free Rings and Their Relations. 2nd Ed. Academic Press, London-New York, 1985. MR 87e: 16006

4. W. Dicks and J. Lewin, A Jacobian conjecture for free associative algebras. Comm. Algebra 10 (1982), 1285-1306. MR 83j: 16046

5. R. H. Fox, Free differential calculus. I. Derivations in free group rings. Ann. of Math. (2) 57 (1953), 547-560. MR 14:843d

6. H. Matsumura, Commutative Algebra. W. A. Benjamin Co., New York, 1970. MR 42:1813

7. A. A. Mikhalev and A. A. Zolotykh, Rank of an element of the free Lie ( $p$-) superalgebra. Doklady Akad. Nauk 334 (1994), no. 6, 690-693 (in Russian; English translation: Russian Acad. Sci. Dokl. Math. 49 (1994), no. 1, 189-193). MR 95a:17009

8. A. A. Mikhalev and A. A. Zolotykh, Rank and primitivity of elements of free color Lie (p-) superalgebras. Internat. J. Algebra and Comput. 4 (1994), 617-655. MR 95k:17003

9. C. Reutenauer, Applications of a noncommutative Jacobian matrix. J. Pure Appl. Algebra 77 (1992), 169-181. MR 93a:16021

10. C. Reutenauer, Free Lie Algebras. Clarendon Press, Oxford, 1993. MR 94j:17002

11. A. H. Schofield, Representations of Rings over Skew Fields. London Math. Soc. Lecture Note Ser. 92 (1985). MR 87c:16001

12. A. I. Shirshov, Subalgebras of free Lie algebras. Mat. Sb. 33 (1953), 441-452 (in Russian). MR 15:596d

13. V. Shpilrain, On generators of $L / R^{2}$ Lie algebras. Proc. Amer. Math. Soc. 119 (1993), 1039-1043. MR 94a: 17002

14. V. Shpilrain, On monomorphisms of free groups. Arch. Math., 46 (1995), 465-470. CMP 95:12

15. U. U. Umirbaev, Partial derivations and endomorphisms of some relatively free Lie algebras. Sib. Math. J. 34 (1993), 1161-1170. MR 95d:17002

16. U. U. Umirbaev, Some algorithmic questions of associative algebras. Algebra and Logic 32 (1993), 450-470. MR 95e:03128

17. U. U. Umirbaev, Primitive elements of free groups. Russian Math. Surveys 49 (1994), no. 2, 184-185. CMP 94:14

18. U. U. Umirbaev, On the rank of elements of free groups. Fundamentalnaya i Prikladnaya Matematika 2 (1966), 313-315. (Russian)

19. A. V. Yagzhev, Algorithmic problem of recognizing automorphisms among endomorphisms of free associative algebras of finite rank. Sib. Math. J. 21 (1980), 142-146. MR 81d:08006

Department of Mechanics and Mathematics, Moscow State University, Moscow 119899 , Russia

E-mail address, A. A. Mikhalev: aamikh@cnit.math.msu.su

Fakultät für Mathematik, Ruhr-Universität Bochum, 44780 Bochum, Germany

Current address: Department of Mathematics, University of California, Santa Barbara, California 93106

E-mail address, Vladimir E. Shpilrain: shpil@math.ucsb.edu

Department of Mechanics and Mathematics, Moscow State University, Moscow 119899, Russia

E-mail address, A. A. Zolotykh: zolotykh@cnit.math.msu.su 